\title{
A SUFFICIENT CONDITION FOR POSITIVITY OF POLYNOMIAL FORMS ${ }^{1}$
}

\author{
F. E. CLARK
}

1. Introduction. In seeking conditions that certain algebraic expressions, primarily polynomials, in $n$ indeterminates be positive, a sufficient condition was obtained when the values assigned to the indeterminates are positive numbers. The result was obtained through the use of Muirhead's theorem as given in $\$ 4$. The methods used in obtaining the result have other possible applications, including an economic one, as given at the end of $\$ 2$.

2. A distribution over a partially ordered system. In the plane, consider a finite set of points $F_{i}(i=1, \cdots, s)$. Partially order $[1, \S 1]$ the set and represent the relation $F_{j} \leqq F_{i}$ by a vector from $F_{i}$ to $F_{j}$. Denote the result by $S$. With each point $F_{i}$ associate a real number $f_{i}$, to be called the supply, and thus get a distribution $(M \mid S)$, which is excessive, balanced, or deficient at $F_{i}$ according as $0<f_{i}, 0=f_{i}$, or $f_{i}<0$.

If $j \neq i$, define $F_{j}<F_{i}$ to mean $F_{j} \leqq F_{i}$ together with $f_{j}<0<f_{i}$. Also define $F_{i} \prec F_{i}$. Consider a pair of values $i, j$ for which $F_{j} \prec F_{i}$. Select $g_{i j}$ to satisfy

$$
0 \leqq g_{i j} \leqq \min \left(f_{i},-f_{j}\right) .
$$

Change the distribution $(M \mid S)$ into a distribution $\left(M^{\prime} \mid S\right)$ by making the supply $f_{i}-g_{i j}$ at $F_{i}, f_{j}+g_{i j}$ at $F_{j}$, and $f_{k}$ at $F_{k}$ where $k \neq i, j$. The passage from $(M \mid S)$ to $\left(M^{\prime} \mid S\right)$ will be called supplying $F_{j}$ with $g_{i j}$ from $F_{i}$.

If a distribution which is nowhere deficient, that is, has all supplies non-negative, can be found from $(M \mid S)$ by a finite number of supply operations, then $(M \mid S)$ will be called adequate.

If $K$ is a subset of $S$, the notation

$$
R(K)=\sum_{K} f_{i}
$$

will be used for the sum of the supply of $K$.

A subset $K$ of $S$ will be called complete if $F_{j} \in K$ together with $F_{j} \leqq F_{i}$ implies $F_{i} \in K$. If $K$ is complete, $R(K)$ is called a reserve for $(M \mid S)$. There is no essential restriction and considerable economy is

Received by the editors June 12, 1951 and, in revised form, February 25, 1952.

1 The content of this paper is taken from a doctoral thesis presented in the Graduate School of Duke University, June, 1948. 
involved if reserves are computed only for every complete subset whose graph is connected.

If $K$ is any subset of $S$, the subset $K^{\prime}$ consisting of all points $F_{j} \in K$ together with all points $F_{i}$ for which $F_{j} \leqq F_{i}$ will be called the completion of $K$. Then $K \subset K^{\prime}$ and $K^{\prime}$ is complete.

Theorem 2.1. A distribution is adequate if and only if all its reserves are non-negative.

The necessity of the condition follows from the observation that in the passage to a nowhere deficient distribution, all elements of a complete subset must be supplied from elements of that set.

To prove the sufficiency, consider the same points partially ordered with respect to $\prec$. This amounts to modifying the graph by erasing every vector which either starts at a point with nonpositive supply or ends at a point with non-negative supply. The resulting system $S^{*}$ consists entirely of maximal points with $0 \leqq f_{i}$ and minimal points with $f_{i} \leqq 0$. There may, of course, be isolated points, which are both maximal and minimal.

Every operation of supply which can be executed in $(M \mid S)$ can also be executed in $\left(M \mid S^{*}\right)$ and conversely. Consequently $(M \mid S)$ is adequate if and only if $\left(M \mid S^{*}\right)$ is adequàte.

If a subset is complete in $S$, it is also complete in $S^{*}$, but not conversely.

Suppose $K$ is complete in $S^{*}$ and consider its completion $K^{\prime}$ in $S$. If $K^{\prime}-K$ is vacuous or consists only of points with nonpositive supply, then

$$
0 \leqq R\left(K^{\prime}\right)=R(K)+R\left(K^{\prime}-K\right) \leqq R(K) .
$$

If $K^{\prime}-K$ contains points $F_{i}$ with $0<f_{i}$, then every point $F_{j} \in K$ for which $F_{j} \leqq F_{i}$ must have $0 \leqq f_{j}$. Removal of all such points $F_{j}$ from $K$ leaves a set $\bar{K}$ which is complete in $S^{*}$ and

$$
0 \leqq R(\bar{K})<R(\bar{K})+R(K-\bar{K})=R(K) .
$$

Thus the reserves of $S^{*}$ are non-negative when those of $S$ are.

Adjust the notation so that $0 \leqq f_{i}$ or $f_{i}<0$ according as $i<r$ or $r \leqq i$ and so that $F_{r} \leqq F_{1}$. Let non-negative $g_{i j}$ be the amount to be supplied $F_{j}$ from $F_{i}$ for $1 \leqq i<r \leqq j \leqq s$. In this way an unknown $g$ is associated with each vector in $S^{*}$.

Since no complete subset contains $F_{r}$ without containing $F_{1}$, the conditions that $\left(M \mid S^{*}\right)$ have non-negative reserves fall into two categories, namely, those of the type

$$
0 \leqq f_{1}+A
$$


and those of the types

$$
0 \leqq f_{1}+f_{r}+B, 0 \leqq C,
$$

where $A, B, C$ are sums involving neither $f_{1}$ nor $f_{r}$.

Set

$$
g_{i r}=\min \left(f_{1},-f_{r}, f_{1}+A\right) .
$$

Change the supply at $F_{1}$ to $f_{1}-g_{1 r}$, change that at $F_{r}$ to $f_{r}+g_{1 r}$, and erase the vector from $F_{1}$ to $F_{r}$ to get a new distribution $\left(M^{*} \mid S^{* *}\right)$. The conditions that $\left(M^{*} \mid S^{* *}\right)$ have non-negative reserves are contained among relations of the types

$$
\begin{aligned}
& 0 \leqq\left(f_{1}-g_{1 r}\right)+A, \\
& 0 \leqq\left(f_{1}-g_{1 r}\right)+\left(f_{r}+g_{1 r}\right)+B, 0 \leqq C,
\end{aligned}
$$

which are implied by (2.1), (2.2), (2.3). Hence, $\left(M^{*} \mid S^{* *}\right)$ has nonnegative reserves.

The operation can be repeated until all vectors have been erased. The final system then consists entirely of isolated points and the supply at each point is non-negative. This completes the proof.

For an economic application, suppose the countries $F_{i}$ form an economic union. Read the symbol "ฏ" as "can import goods from," the ordering being assigned by the rules of the union. Let $a_{i}$ be the number of tons of coal on hand, $b_{i}$ the number of tons needed, and $f_{i}$ the difference $a_{i}-b_{i}$. Theorem 2.1 tells whether the distribution of coal is adequate.

3. Non-negative combinations of positive functions. Consider the expression

$$
F=f_{1} F_{1}+\cdots+f_{8} F_{s}
$$

where $f_{1}, \cdots, f_{s}$ are real numbers and $F_{1}, \cdots, F_{s}$ are functions whose values are positive and satisfy certain inequalities

$$
F_{j}(x) \leqq F_{i}(x)
$$

for all $(x)$ in a fixed $n$-dimensional Euclidean domain $V$. Relations (3.2) partially order the $F$ 's. If the reserves of the distribution defined in $\$ 2$ are non-negative, the function $F$ is non-negative in $V$. If one reserve is positive, then $F$ is positive.

Using the distribution $\left(M \mid S^{*}\right)$ and the notation of $\S 2$, we have

$$
F=\left(f_{1}-g_{1 r}\right) F_{1}+\left(f_{r}+g_{1 r}\right) F_{r}+g_{1 r}\left(F_{1}-F_{r}\right)+\cdots .
$$

Since $0 \leqq g_{1 r}\left(F_{1}-F_{r}\right)$, 


$$
\left(f_{1}-g_{1 r}\right) F_{1}+\left(f_{r}+g_{1 r}\right) F_{r}+\cdots \leqq F .
$$

When all the supplies have been made non-negative, we have

$$
f_{1}^{*} F_{1}+\cdots+f_{r}^{*} F_{r} \leqq F,
$$

so that $F$ is non-negative, and if one $f^{*}$ is positive, so is $F$.

4. Symmetric forms. Muirhead's theorem [3], also quoted in [2, $\$ 2.18]$ states that if all coordinates of $(x)$ are positive, then

$$
\omega(x ; i) \leqq \omega(x ; j)
$$

if and only if $(i) \leqq(j)$, where

$$
\omega(x ; i)=\frac{1}{n !} \sum_{(x)} x_{1}^{i_{1}} x_{2}^{i_{2}} \ldots x_{n}^{i_{n}}
$$

the summation being taken over all permutations of $(x)$ for fixed $(i)$. The notation $(i) \leqq(j)$ means

$$
\sum_{p=0}^{n-1} i_{n-p}=\sum_{p=0}^{n-1} j_{n-p}, \quad \sum_{p=0}^{k-1} i_{n-p} \leqq \sum_{p=0}^{k-1} j_{n-p} \quad(k=1,2, \cdots, n-1),
$$

where $i_{1} \leqq i_{2} \leqq \cdots \leqq i_{n}, j_{1} \leqq j_{2} \leqq \cdots \leqq j_{n}$. This is a partial ordering of the indices $(i)$ and hence of the functions $\omega(x ; i)$ of same degree.

Every symmetric form of degree $p$ in $n$ indeterminates can be written

$$
\phi_{n}^{p}(x)=\sum_{i_{1}+\cdots+i_{n}=p} f_{(i)} \omega(x ; i) .
$$

The result of the preceding section gives sufficient conditions that $\phi$ be non-negative or positive.

If, as for a quadratic form, there are just two terms in $\phi$ and (i) $\leqq(j)$, then conditions are

$$
0 \leqq f_{(j)}, \quad 0 \leqq f_{(i)}+f_{(j)} .
$$

The symmetric cubic form $\phi_{n}^{3}$ has three terms corresponding to $\left(i_{1}\right)=(1,1,1,0, \cdots, 0),\left(i_{2}\right)=(2,1,0, \cdots, 0),\left(i_{3}\right)=(3,0, \cdots, 0)$ and can be written

$$
\dot{\phi}_{n}^{3}(x)=f_{1} \omega_{1}+f_{2} \omega_{2}+f_{3} \omega_{3} .
$$

The sufficient conditions that it be non-negative are

$$
0 \leqq f_{1}+f_{2}+f_{3}, \quad 0 \leqq f_{2}+f_{3}, \quad 0 \leqq f_{3} .
$$

These conditions, however, are not necessary. We have 


$$
\begin{gathered}
\phi_{n}^{3}(x)=\left(f_{1}+f_{2}+f_{3}\right) \omega_{1}+\left(f_{2}+2 f_{3}\right)\left(\omega_{2}-\omega_{1}\right)+f_{3}\left(\omega_{1}-2 \omega_{2}+\omega_{3}\right), \\
0 \leqq \omega_{1}-2 \omega_{2}+\omega_{3}
\end{gathered}
$$

so that another set of sufficient conditions is

$$
\text { (4.3) } \quad 0 \leqq f_{1}+f_{2}+f_{3}, \quad 0 \leqq f_{2}+2 f_{3}, \quad 0 \leqq f_{3} .
$$

Conditions (4.2) imply (4.3), but not conversely.

\section{REFERENCES}

1. Garrett Birkhoff, Lattice theory, rev. ed., Amer. Math. Soc. Colloquium Publications, vol. 25, 1948.

2. G. H. Hardy, J. E. Littlewood, and G. P6lya, Inequalities, Cambridge University Press, 1934.

3. R. F. Muirhead, Some methods applicable to identities and inequalities of symmetric algebraic functions of $n$ letters, Proceedings of the Edinburgh Mathematical Society vol. 21 (1903) pp. 144-157.

DUKE UNIVERSITY 